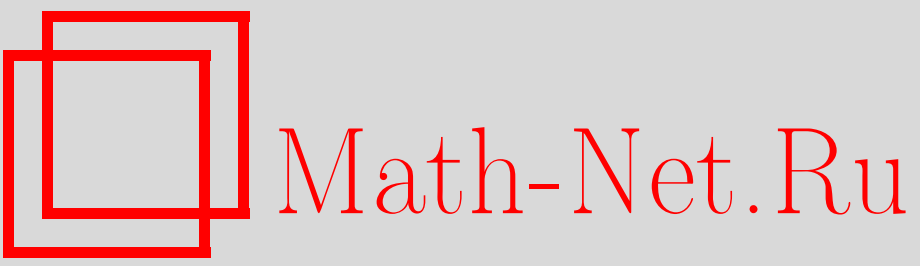

В. В. Дубровский, Глобальные представления ядра резольвенты несамосопряженного оператора по Келдышу, УМН, 1996, том 51, выпуск 3, 199-200

DOI: https://doi.org/10.4213/rm983

Использование Общероссийского математического портала Math-Net.Ru подразумевает, что вы прочитали и согласны с пользовательским соглашением

http://www.mathnet.ru/rus/agreement

Параметры загрузки:

IP: 34.227 .88 .159

26 апреля 2023 г., 13:11:08 


\title{
ГЛОБАЛЬНЫЕ ПРЕДСТАВЛЕНИЯ ЯДРА РЕЗОЛЬВЕНТЫ НЕСАМОСОПРЯЖКЕННОГО ОПЕРАТОРА ПО КЕЛДЫШУ
}

\author{
В. В. ДуБровский
}

Пусть $T$ - полуограниченный снизу, дискретный оператор в $L_{2}(D)$, где $D$-компакт в $\mathbb{R}^{N}$, $N \geqslant 1 ;\left\{\lambda_{n}\right\}_{n=1}^{\infty}-$ все собственные числа оператора $T$, занумерованные в порядка возрастания их величин с учетом кратности; $\left\{v_{n}\right\}_{n=1}^{\infty}-$ собственные ортонормированные векторы оператора $T$, отвечающие собственным значениям $\lambda_{n}$, т.е. $T v_{n}=\lambda_{n} v_{n},\left(v_{n}, v_{m}\right)=\int_{D} v_{n}(x) \overline{v_{n}(x)} d x=$ $\delta_{m n}$. Предположим, что $\lambda_{n}=C n^{\alpha}+o\left(n^{\alpha}\right)$, где $C>0, \alpha>1$. Хорошо известны (см. [2]):

УтвеРЖДЕнИЕ 1. Существует подпоследовательность $\left\{n_{m}\right\}_{m=1}^{\infty}$ натуральных чисел такая, что

$$
\lambda_{n_{m}+1}-\lambda_{n_{m}} \geqslant \operatorname{const}\left(n_{m}\right)^{\alpha-1}>0 .
$$

УТВЕРЖДЕНИЕ 2. Если $\left|n-n_{m}\right| \geqslant C_{1} n_{m}$, әде $C_{1}>0$ - сколь угодно малое фиксированное число, то

$$
\left|\lambda_{n}-\lambda_{n_{m}}\right| \geqslant C_{2} \max \left\{n, n_{m}\right\}^{\alpha},
$$

әде $C_{2}>0$ зависит от $C_{1}$

Пусть $P$ - вообще говоря, несамосопряженньй ограниченный оператор в $L_{2}(D) ; \mu_{n}$ - все собственные числа оператора $T+P$, взятые с учетом алгебраической кратности и занумерованные в порядке возрастания их действительных частей.

Возьмем для данного $\mu_{n}$ произвольную каноническую систему (см. [1]) собственных и присоединенных векторов оператора $T+P$. Присоединим к ней надлежаще выбранную систему собственных и присоединенных векторов оператора $T+P$, отвечающую собственному числу $\bar{\mu}_{n}$ оператора $T+P$, такую, что ядро резольвенты $K_{T+P}(x, y, \lambda)$ оператора $T+P$ имеет с точностью до ограниченного остатка в $L_{\infty}(D)$ вид суммы простейших дробей вблизи $\mu_{n}$, выраженных через эти канонические системы (см. [1]). Обозначим $O_{m}=\left\{\lambda:|\lambda|=\frac{1}{2}\left(\lambda_{n_{m}}+\lambda_{n_{m}+1}\right)\right\}$ окружности с центром в начале координат радиуса $\frac{1}{2}\left(\lambda_{n_{m}}+\lambda_{n_{m}+1}\right)>0$. Если для некоторого натурального $q$ (см. [3])

$$
\varlimsup_{m \rightarrow \infty} \frac{\max _{x \in O_{m}}\left\|K_{T+P}(x, y, \lambda)\right\|_{L_{2}(D \times D)}}{\left(\frac{1}{2}\left(\lambda_{n_{m}}+\lambda_{n_{m}+1}\right)\right)^{q}}<\infty,
$$

то ядро $K_{T+P}(x, y, \lambda)$ резольвенты оператора $T+P$ представимо в виде суммы простейших дробей без остатка, просуммированных в $L_{2}(D \times D)$. Но из тождества Гильберта для ядер резольвенты вытекает, что неравенство (1) достаточно доказать для ядра $K_{T}(x, y, \lambda)$ резольвенты оператора $T$ на окружностях $O_{m}$. Тогда

$$
K_{T}(x, y, \lambda)=\sum_{i=1}^{\infty} \frac{v_{i}(x) \overline{v_{i}(y)}}{\lambda_{i}-\lambda},
$$

следовательно (см. [2]), имеем

$$
\begin{aligned}
\left\|K_{T}(x, y, \lambda)\right\|_{L_{2}(D \times D)} & \leqslant \text { const } \sum_{i=1}^{\infty} \frac{1}{\left|\lambda_{i}-\lambda\right|} \\
& \equiv \sum_{i=1}^{n_{m}-\left[C_{1} n_{m}\right]-1}+\sum_{i=n_{m}-\left[C_{1} n_{m}\right]}^{n_{m}+\left[C_{1} n_{m}\right]}+\sum_{i=n_{m}+\left[C_{1} n_{m}\right]+1} \\
& \equiv J_{1}+J_{2}+J_{3} .
\end{aligned}
$$


Оценим каждое из $J_{j}, j=\overline{1,3}$ : пользуясь утверждением 2 , имеем

$$
J_{1}=\mathrm{const} \sum_{i=1}^{n_{m}-\left[C_{1} n_{m}\right]-1}\left|\lambda_{i}-\lambda\right|^{-1} \leqslant \mathrm{const} \sum_{i=1}^{n_{m}-\left[C_{1} n_{m}\right]-1} \frac{\text { const }}{\left(n_{m}\right)^{\alpha}} \leqslant \operatorname{const}\left(n_{m}\right)^{1-\alpha} .
$$

Далее, из утверждения 1 вытекает, что

$$
J_{2} \leqslant \text { const } \sum_{i=n_{m}-\left[C_{1} n_{m}\right]}^{n_{m}+\left[C_{1} n_{m}\right]} \text { const } n_{m}^{1-\alpha} \leqslant \text { const } n_{m}^{2-\alpha} .
$$

Точно так же, как $J_{1}$, оценивается $J_{3}$ при $\alpha>1$. Следовательно, при $\lambda \in O_{m}$

$$
\left\|K_{T}(x, y, \lambda)\right\|_{L_{2}(D \times D)} \leqslant \operatorname{const}\left(n_{m}\right)^{2-\alpha},
$$

где const не зависит от $m$. Тогда

$$
\frac{\max _{O_{m}}\left\|K_{T}(x, y, \lambda)\right\|_{L_{2}(D \times D)}}{n_{m}^{\alpha q}} \leqslant \text { const } n_{m}^{2-(q+1) \alpha}<\infty
$$

при $q>1$. Следовательно, доказана следующая

Теорема 3. Ядро резольвенты оператора $T+P$ представимо при $\alpha>1$ в виде суммы простейших дробей, выражсенных через выбранные по М. В. Келдышу канонические системы собственных и присоединенных векторов операторов $T+P u T+P^{*}$, причем сходимость әтой суммы понимается со скобками со значениями в $L_{2}(D \times D)$.

Аналогично доказывается с помошью теоремы Рисса-Торина

Теорема 4. Если собственные ортонормированные векторы $v_{n}(x)$ оператора $T$ равномерно ограничены в $L_{\infty}(D)$, то ядро резольвенты оператора $T+P$ представимо при $\alpha>1$ в виде суммы простейших дробей, выраженных через выбранные по М. В. Келдышу канонические системы собственных и присоединенных векторов операторов $T+P$ и $T+P^{*}$, причем сходимость әтой суммы понимается со скобками со значениями в $L_{p}(D \times D) \quad \forall p \geqslant 2$.

ЗАмечАниЕ 5 . Класс эллиптических псевдодифференциальных операторов, к которым применима теорема 3 , достаточно широк. Сюда входят все операторы вида $(-\Delta)^{\alpha}+P, \alpha>1$, где $\Delta$ - оператор Лапласа, заданный на замкнутом двумерном римановом многообразии, а $P$ - вообще говоря, комплексная существенно ограниченная измеримая на многообразии функция.

ЗАмечАнИЕ 6 . Суммируемость ядра резольвенты оператора $T+P$ со скобками можно было рассматривать в $L_{p}(D \times D), p \geqslant 1$.

ЗАмечание 7 . Оператор $P$ для простоты взят ограниченным, а не подчиненным, в том или ином смысле малости, оператору $T$.

ЗАмечАнИЕ 8. Частные случаи теорем 3 и 4 рассмотрены в работах [4], [5].

Автор выражает благодарность В. А. Ильину, В. А. Садовничему, В. Ф. Кравченко, Е. И. Моисееву, Л. М. Велюсу и Т.К. Плышевской за обсуждение тематики данной работы.

\section{СПИСОК ЛИТЕРАТУРЫ}

[1] Келдыш М. В. // ДАН СССР. 1951. Т. 77. С. 11-14. [2] Дубровский В. В. // Дифф. уравнения. 1990. Т. 26. № 12. С. 2046-2051. [3] Маркушевич А. И. Краткий курс теории аналитических функций. М.: Наука, 1966. [4] Дубровский В. В., Кравченко В. Ф. // УМН. 1994. Т. 49. № 1. С. 211-212. [5] Дубровский В. В. // УМН. 1994. Т. 49. № 4. С. 163-164. 